\title{
Structure and Dynamics of Annexin 12 Bound to a Planar Lipid Bilayer
}

\author{
T. Risse* and W. L. Hubbell ${ }^{\dagger}$ \\ Jules Stein Eye Institute and Department of Chemistry and Biochemistry, University of California, \\ Los Angeles, California 90095, USA \\ J. M. Isas and H. T. Haigler \\ Department of Physiology and Biophysics, University of California, Irvine, California 92697, USA
}

(Received 10 March 2003; published 29 October 2003)

\begin{abstract}
Site directed spin labeling is used to investigate the protein annexin 12 absorbed on a single planar phospholipid bilayer of approximately $2-3 \mathrm{~cm}^{2}$. Electron paramagnetic resonance spectra of nitroxide side chain at several topological sites reveal a conserved tertiary fold of the protein in the absorbed state, in agreement with earlier diffraction results. The angular dependent spectra of the twodimensional microcrystals are shown to provide information on the degree of ordering of spin labels in a $\alpha$-helix and in turn on the orientation of the $\alpha$-helix with respect to the surface.
\end{abstract}

DOI: 10.1103/PhysRevLett.91.188101

PACS numbers: $87.14 . \mathrm{Ee}, 87.15 . \mathrm{Kg}, 87.64 . \mathrm{Hd}$

There is currently intense interest in the interaction of proteins with surfaces and in the properties of the surface that determine the interaction. Different surfaces have been developed as models for surfaces in living organisms, and numerous analytical tools have been used to explore their properties (e.g., [1,2]). However, little is known about the structure and dynamics of the adsorbed proteins, because the number of experimental techniques to address this issue is limited. Spectroscopic methods such as IR, circular dichroism, or fluorescence spectroscopy provide only global information on the secondary structure of absorbed proteins or probe the properties of a specific chromophore (e.g., [3,4]), but sequence-specific information on both secondary and tertiary structure is essential to determine the state of the protein on the surface. Such information is traditionally the domain of diffraction techniques, but the necessity of a crystalline arrangement of molecules restricts the applicability of the methods to a few cases (e.g., [5-7]).

In this Letter, an electron paramagnetic resonance (EPR) spectroscopic method is employed that can provide sequence-specific secondary and tertiary structure (in the following we will use the term "structure" as a synonym for the tertiary fold of the protein) without the need for two-dimensional crystals. The method, called site-directed spin labeling (SDSL), involves the introduction of a nitroxide side chain at selected sites by mutation of the native amino acid to cysteine. Subsequently, the cysteine is modified by the reaction with a highly specific nitroxide reagent to introduce a paramagnetic side chain designated R1 (Fig. 1). The EPR spectrum of the labeled protein reflects the mobility of the R1 side chain, and studies in a number of proteins have shown that the side chain mobility is uniquely determined by the local structure of the protein and the backbone dynamics. Thus, the EPR spectra for a set of R1 side chains at specific sites throughout the protein provide a "fingerprint" for the global protein structure and can be used to determine sequence-specific secondary structure, reveal tertiary organization, monitor structural changes related to protein function, and map the backbone dynamics [8-11].

To evaluate SDSL as a strategy for monitoring the structure of proteins on surfaces, annexin 12 absorbed on a single supported lipid bilayer is used as a model system. Annexins are a family of proteins that share the common property of reversible $\mathrm{Ca}^{2+}$-dependent binding to membranes containing phosphatidylserine, with little change in the internal structure [7,12]. When adsorbed on a bilayer, annexin 12 forms large two-dimensional crystals and offers one of the rare opportunities to compare the results obtained with those of diffraction and highresolution microscopic techniques [7,13]. However, it must be emphasized that a crystalline arrangement of the molecules is not mandatory for the SDSL/EPR strategy used here.

In the present experiments, a binary mixture of 3:1 mol ratio 1-Palmitoyl-2-Oleoyl-sn-Glycero-3Phosphocholine (POPC), 1-Palmitoyl-2-Oleoyl-snGlycero-3-[Phospho-L-Serine] (POPS) was used to form a negatively charged bilayer supported on a quartz surface. The formation of the oriented film was first verified by doping the mixture with $2.5 \mathrm{~mol} \%$ of a spin labeled PC analog (5-doxylPC). Previous studies have shown that such spin labeled lipids have approximate axial magnetic symmetry in fluid lipid bilayers due to

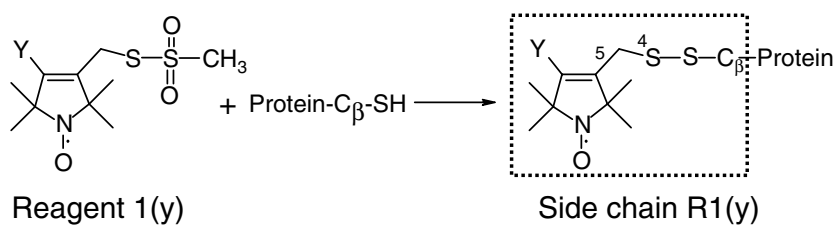

FIG. 1. Reaction of a methanethiosulfonate spin label reagents 1(a) or 1(b) with a protein sulfhydryl to generate the corresponding side chain designated $\mathrm{R} 1(\mathrm{a})(Y=\mathrm{H})$ or $\mathrm{R} 1(\mathrm{~b})\left(Y=\mathrm{CH}_{3}\right)$. 
rapid rotational diffusion about the long axis of the molecule [14]. The spatial anisotropy of the magnetic interaction allows a determination of the orientation of ordered bilayer by angular dependent measurements. The changes of the angular resolved EPR spectra indicate the expected orientation of the bilayer on the quartz surface (data not shown).

To monitor the structure of annexin XII, residues 5, 148,154 , and 213 were selected for the introduction of spin labels (Fig. 2).

These residues are located at distinct topographical regions in the molecule and have been previously investigated for annexin in solution $[16,17]$. The corresponding EPR spectra, reproduced in Fig. 3(a), are consistent with, and diagnostic for, the native structure of the protein as revealed by the crystal structure [15]. For example, residue 5 is located close to the end of the $N$-terminal tail, a sequence that is statically disordered in the crystal structure. For annexin in solution, the narrow linewidths of $\mathrm{R} 1$ (a) at this position reveal a rapid, essentially isotropic motion, corresponding to a dynamically disordered sequence. In the crystal structure, residues 148 and 154 are located on opposing surfaces of the same $\alpha$-helix (helix $E$, Fig. 2), with 148 facing the solvent and 154 being completely solvent inaccessible, facing the solidlike interior of the protein. As expected for these locations, the broad EPR "powder" spectrum of 154 reflects a highly immobilized state, while that of 148 reflects an intermediate degree of mobility typical of R1(a) at solventexposed helix sites [18]. As shown in Fig. 2, 148 lies adjacent to the loop connecting helices $D$ and $E$, one of several sites in annexin believed to be a partner in cocoordinating $\mathrm{Ca}^{2+}$ with negative charges in the lipid bilayer [12]. Finally, 213 is located at a solvent-exposed site at the $C$-terminal end of an $\alpha$-helix. The EPR spectrum reflects a relatively mobile state characteristic of

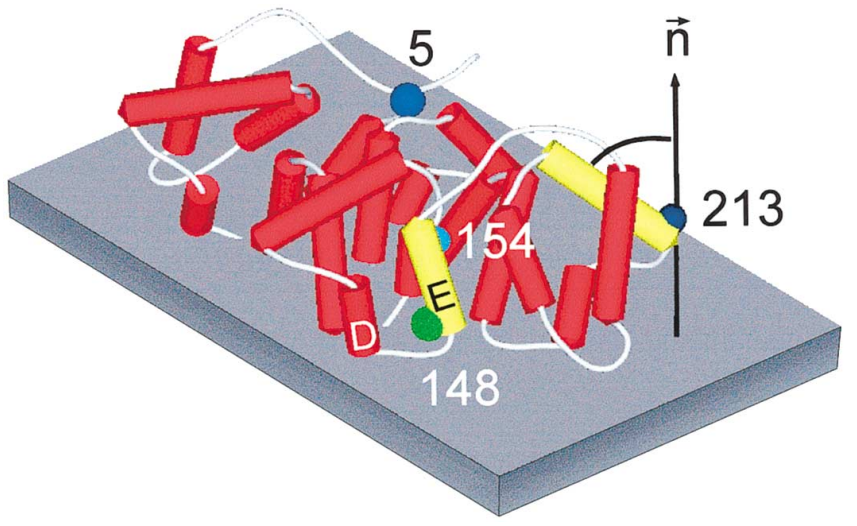

FIG. 2 (color). Structural model of annexin 12 [15]. The locations of residues 5, 148, 154, and 213 are indicated by spheres on the alpha carbon atoms. The surface represents the headgroup region of the bilayer and was selected to pass through the $\mathrm{Ca}^{2+}$ ions in the crystal structure which are the lipid binding sites. such sites, due in part to contributions from backbone motions [18].

Spin labeled annexins absorb reversibly to the supported bilayer in a $\mathrm{Ca}^{2+}$-dependent fashion at $p \mathrm{H} 7.4$ to give the EPR spectra shown in Fig. 3(b). The surface density of annexin in the film of 213R1(a), estimated by double integration of the EPR spectrum, is similar to that in the $2 \mathrm{D}$ crystals formed by the homologous annexin 5 [13]. Remarkably, the spectra of R1(a) at each site in the adsorbed state are very similar to those in solution. Because the motions of the side chains are strongly dependent on structure, and because the residues are distributed throughout the molecule, this result strongly argues that the basic fold of the molecule is preserved upon adsorption to lipid bilayers under physiological conditions. Apparently, neither lipid-protein nor proteinprotein interactions in the lattice change the fold of the protein in the regions sampled by the spin labels. This strengthens similar conclusions from previous electron crystallographic studies of the homologous annexin 5 adsorbed on monolayers of the same lipid composition used here, but imbedded in an anhydrous negative stain and maintained in a high vacuum (see, e.g., Refs. [7,12]).

If the nitroxide side chain has a unique time-averaged conformation, and the motion is sufficiently constrained so that the $A$ and $g$ anisotropy is not averaged, the EPR spectra should depend on the orientation of the bilayer with respect to the external field. Of the sites investigated with R1(a), only the spectrum of 213 shows an angular
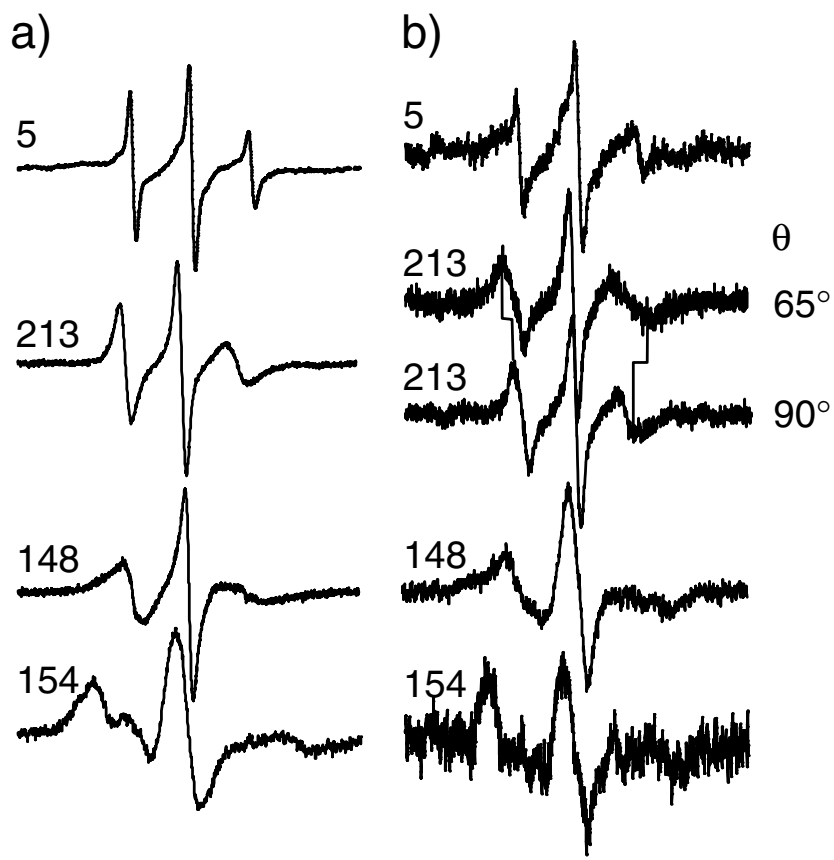

FIG. 3. EPR spectra of annexin 12 with R1(a) at the indicated sites. (a) In solution; (b) adsorbed to a single planar bilayer. R1(a) at sites 5, 148, and 154 are angle independent. For 213 spectra are shown for two values of $\theta$, the polar angle between the field and the bilayer normal. 
dependence (Fig. 2), although it is weak due to the high mobility of the nitroxide and the concomitant averaging of the $A$ and $g$ tensors. Similarly, the lack of angular dependence of R1(a) at site 5 can be attributed to the rapid, essentially isotropic, motion. Interestingly, R1(a) at both 148 and 154 show no angular dependence, even though their hindered motions cannot be sufficient to completely average the magnetic anisotropies. In these cases, the interactions of the side chain with the environment must lead to static disorder, i.e., the interactions must give rise to multiple conformations of the side chain with different orientations of the nitroxide ring.

Previous studies of the R1 side chain at solventexposed helix surface sites, such as 213 , have revealed that internal side chain motions are dominated by torsional oscillations about bonds $X_{4}$ and $X_{5}$ (Fig. 1). The weak angular dependence of 213R1(a) noted in Fig. 3(b) is due to this motion coupled with fluctuations in the protein backbone. It has been shown recently that a 4- $\mathrm{CH}_{3}$ substitution on the five-membered nitroxide ring [side chain R1(b), Fig. 1] leads to a sharply decreased amplitude of the $X_{4} / X_{5}$ motion [19]. The effect can be

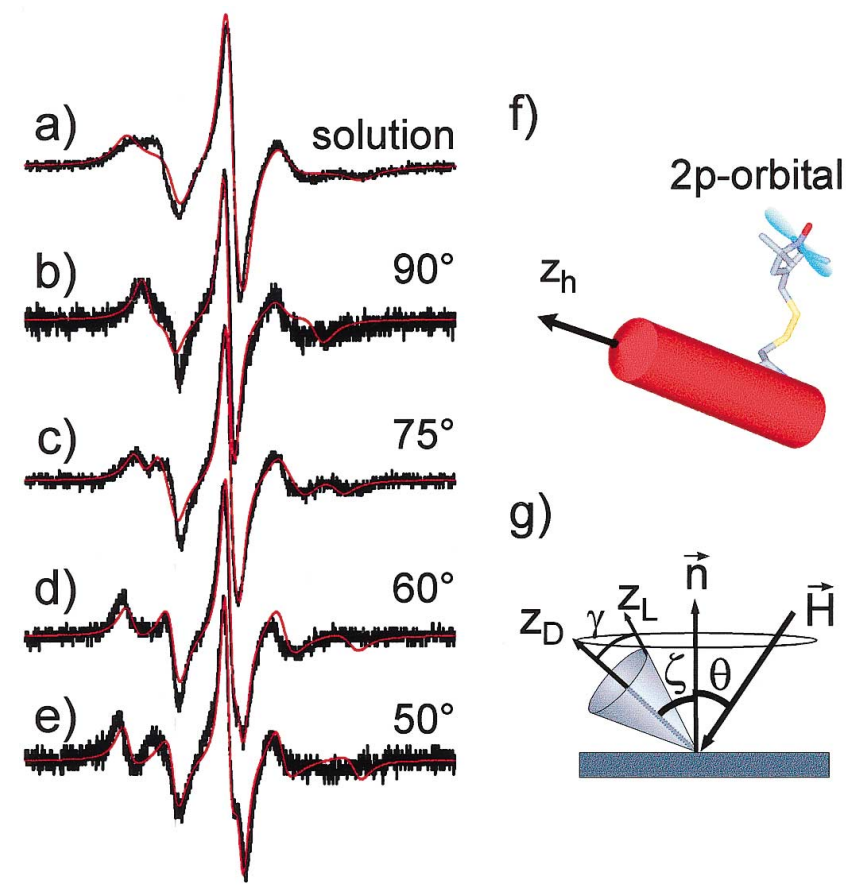

FIG. 4 (color). EPR spectra of annexin 12 with R1(b) at 213 and models for the R1 side chain and its motion on helices. (a) In solution. (b) -(e) Adsorbed to a single planar bilayer oriented at the indicated polar angle $(\theta)$ with respect to the field. The red traces are fits to the spectra (cf. text). (f) Model of the 213R1(a) side chain on a helix showing the orientation of the $2 p$ orbital of the nitrogen and the helical axis, $Z_{h}$. (g) Definition of the axis and angles used: $Z_{L}$ is the (instantaneous) axis of the $p$ orbital of the nitroxide, which moves within a cone; $Z_{D}$ is the symmetry axis of the anisotropic motion (the director axis); $\vec{n}$ is the bilayer normal; $\vec{H}$ is the direction of the static magnetic field; $\gamma \angle\left(Z_{D}, Z_{L}\right) ; \zeta \angle\left(Z_{D}, \vec{n}\right) ; \theta \angle(\vec{n}, \vec{H})$. seen by a comparison of the spectra of 213R1(a) [Fig. 3(a)] and 213R1(b) [Fig. 4(a)] recorded in solution. Thus, it is expected that the R1(b) side chain should have increased ordering with respect to the protein. To investigate this prediction, the orientation dependence of 213R1(b) absorbed to planar bilayers was examined, and the EPR spectra are shown in Figs. 4(b)-4(e). It is evident that the angular anisotropy of 213R1(b) is significantly greater than that for 213R1(a) shown in Fig. 3(b).

The average orientation of the nitroxide relative to the surface can be extracted from the data in Fig. 4 by fitting the spectra to a model for the nitroxide motion. Previous work has shown that the motion of the nitroxide can be described by a single order parameter $(S)$ and a correlation time of 1-2 nsec [19] [Figs. 4(f) and 4(g)]. This motion is sufficiently fast to validate the use of an effective Hamiltonian approach for an approximate simulation of the spectra $[14,20]$. This approach is used here with nuclear spin state-dependent linewidths to account for residual dynamical effects neglected in this approximation [19,21]. Thus, individual spectra were calculated for an anisotropic motion characterized by a single order parameter $S\left[S=-1 / 2<\left(3 \cos ^{2} \gamma-1\right)>; \gamma \angle\left(Z_{D}, Z_{L}\right)\right.$ [Fig. $4(\mathrm{~g})]$; the brackets indicate the spatial average]. An effective Hamiltonian fit to the spectrum of 213R1(b) in solution is shown in Fig. 4(a) (red trace).

Although annexin forms a two-dimensional lattice on the surface, the molecules are azimuthally disordered due to the presence of crystallites. Thus, spectra for a particular polar angle of the external field relative to the surface were computed by averaging over the azimuthal angle (Fig. 4). Experimental spectra were fit to this model with $S$ and the tilt angle of the director relative to the surface [ $\zeta$ Fig. $4(\mathrm{~g})]$ as fitting parameters. Extensive search in the parameter space reveals a global minimum for $S=0.45$ and $\zeta=40^{\circ}$ [red traces, Figs. 4(a)-4(e), for trace (a) an isotropic distribution of directors was assumed]. The global fit to the spectra is very sensitive to the choice of tilt angle, and changes by $\pm 10^{\circ}$ reduced the quality of the fit considerably.

From models of the R1 side chain on helices based on crystal structures [22] and EPR line shape analysis [19], it was inferred that the average orientation of the nitroxide $p$ orbital should lie roughly perpendicular to the helical axis [Fig. 4(f)]. By modeling the nitroxide side chain into the crystal structure the tilt angle of the $p$ orbital determined by EPR spectroscopy results in a helix tilt angle $\phi$ of $\approx 50^{\circ}$ with respect to the surface. This is in good agreement with the orientation of the helix determined by diffraction experiments (Fig. 2). This result suggests that the angular dependence of the EPR spectra might be used to determine the orientation of secondary structural elements of a protein relative to the surface. This would be extremely useful not only for evaluating the structure of the absorbed protein, but also to monitor rigid body rearrangements during conformational changes. 
In summary, the results presented here show that SDSL is a suitable approach to determine the structure of proteins absorbed at surfaces. In principle, the entire suite of SDSL methods developed for solution structural analysis can be directly employed to analyze their counterparts at interfaces. This includes the determination of sequencespecific secondary structure through solvent accessibility and side chain dynamics, interresidue distance determination using dipolar interactions, and analysis of backbone dynamics. In addition, the demonstration that side chains with hindered internal motions have a net orientation may lead to a new approach to reveal the orientation of helical segments within proteins at surfaces.

Methods.-Specific mutations were introduced into the annexin 12 pSE420-mrp33H plasmid [23] as described previously [24]. The modification of the introduced cysteine with the spin label was performed as described before [16]. Spin labeled mutants are designated by giving the sequence position of the cysteine substitution with a suffix specifying the nitroxide side chain. For example, 148R1(a) is the mutant with side chain R1(a) at site 148 . Samples were prepared in aqueous flat cells (Wilmad WQ $812 \mathrm{~S}$ ). The cell surface was cleaned by rinsing sequentially with chloroform, acetonitrile, ethanol, and water, followed by sonification with detergent and a thorough rinse with water. Prior to use the cells were heated to $650{ }^{\circ} \mathrm{C}$ for at least $24 \mathrm{~h}$. Lipid bilayers consisting of a 3:1 mol ratio of POPC and POPS (Avanti Polar Lipid) were deposited on the inner surface of the flat cell as described in the literature [25]. Briefly, the cleaned flat cell was exposed for $2 \mathrm{~h}$ to an aqueous suspension of small unilamellar lipid vesicles produced by sonication of the lipid mixture suspended in an aqueous buffer [100 $\mathrm{mM}$ (4-(2-hydroxyethyl)-1-piperazineethanesulfonic acid) (Hepes), $100 \mathrm{mM} \mathrm{NaCl}, p \mathrm{H} \mathrm{7.4].} \mathrm{Excess} \mathrm{vesicles} \mathrm{were}$ removed by washing the flat cell with pure buffer. To bind annexin to the bilayer, $1 \mathrm{mM} \mathrm{CaCl}$ was added to the buffer. Excess unbound protein was removed by washing. EPR spectra were recorded with a Varian E 109 EPR spectrometer equipped with a TM 110 resonator. The spectra were corrected by subtracting corresponding background spectra taken after removal of the annexin 12 by washing with an EDTA containing buffer $(100 \mathrm{mM}$ Hepes, $100 \mathrm{mM} \mathrm{NaCl}, p \mathrm{H}$ 7.4, $50 \mathrm{mM}$ EDTA).

We thank Dr. Christian Altenbach for helpful comments on the manuscript. T. R. would like to thank the Deutsche Forschungsgemeinschaft for a research fellowship. This work was supported in part by the NIH Grants No. GM 55651 (H.T. H) and No. EY05216 (W. L. H), the Jules Stein Professorship Endowment, the Bruce Ford Bundy and Anne Smith Bundy Foundation, and the Army Research Office Research Agreement No. Daad1901-1-0522 (W. L. H.).
*Permanent address: Fritz-Haber-Institut der Max-Planck Gesellschaft, Faradayweg 4-6, 14195 Berlin, Germany. Corresponding author. Email address: WLH hubbellw@jsei.ucla.edu

[1] E. Sackmann, Science 271, 43 (1996).

[2] M. Mrksich, Curr. Opin. Colloid Interface Sci. 2, 83 (1997).

[3] L. L. Wood, S. S. Cheng, P. L. Edmiston, and S. S. Saavedra, J. Am. Chem. Soc. 119, 571 (1997).

[4] P. H. Axelsen, B. K. Kaufman, R. N. McElhaney, and R. Lewis, Biophys. J. 69, 2770 (1995).

[5] U. B. Sleytr, M. Sara, D. Pum, and B. Schuster, Prog. Surf. Sci. 68, 231 (2001).

[6] B. Berge, P. F. Lenne, and A. Renault, Curr. Opin. Colloid Interface Sci. 3, 321 (1998).

[7] A. Brisson, G. Mosser, and R. Huber, J. Mol. Biol. 220, 199 (1991).

[8] L. Columbus and W. L. Hubbell, Trends Biochem. Sci. 27, 288 (2002).

[9] W. L. Hubbell, D. S. Cafiso, and C. Altenbach, Nat. Struct. Biol. 7, 735 (2000).

[10] J. B. Feix and C.S. Klug, in Biological Magnetic Resonance, edited by L.J. Berliner (Plenum Press, New York, 1998), Vol. 14, pp. 251-281.

[11] W. L. Hubbell, A. Gross, R. Langen, and M. A. Lietzow, Curr. Opin. Struct. Biol. 8, 649 (1998).

[12] S. Liemann and R. Huber, Cell Mole Life Sci. 53, 516 (1997).

[13] I. Reviakine, W. Bergsma-Schutter, and A. Brisson, J. Struct. Biol. 121, 356 (1998).

[14] W. L. Hubbell and H. M. McConnell, J. Am. Chem. Soc. 93, 314 (1971).

[15] H. Luecke, B. T. Chang, W. S. Mailliard, D. D. Schlaepfer, and H.T. Haigler, Nature (London) 378, 512 (1995).

[16] J. M. Isas, R. Langen, H. T. Haigler, and W. L. Hubbell, Biochemistry 41, 1464 (2002).

[17] R. Langen, J. M. Isas, H. T. Haigler, and W. L. Hubbell (unpublished).

[18] H. S. Mchaourab, M. A. Lietzow, K. Hideg, and W. L. Hubbell, Biochemistry 35, 7692 (1996).

[19] L. Columbus, T. Kalai, J. Jekö, K. Hideg, and W. L. Hubbell, Biochemistry 40, 3828 (2001).

[20] O. H. Griffith and P. C. Jost, in Spin Labeling Theory and Applications, edited by L. J. Berliner (Academic Press, New York, 1976), pp. 454-523.

[21] B. J. Gaffney and H. M. McConnell, J. Magn. Reson. 16, 1 (1974).

[22] R. Langen, K. J. Oh, D. Cascio, and W. L. Hubbell, Biochemistry 39, 8396 (2000).

[23] W.S. Mailliard, H. Luecke, and H.T. Haigler, Biochemistry 36, 9045 (1997).

[24] R. Langen, J. M. Isas, H. Luecke, H. T. Haigler, and W. L. Hubbell, J. Biol. Chem. 273, 22453 (1998).

[25] Y. Barenholz, D. Gibbes, B. J. Litman, J. Goll, T. E. Thompson, and R. D. Carlson, Biochemistry 16, 2806 (1977). 